Talashova Svetlana Vadimovna, PhD, associate professor of a Chair of Pharmacognosia and Pharmaceutical Technology, State Budget Educational Institution of High Professional Education "Yaroslavl State Medical Academy", Ministry of Health, Russian Federation

Address: 150000, Yaroslavl, Revolutsyonnaya str. 5, Tel.: (4852) 30-31-40, e-mail: stalash@rambler.ru

Article received: 25.01.2013, Accepted for publication: 01.02.2013

В настоящее время нарастает частота встречаемости детской неврологической патологии, связанной с выраженными умственными перегрузками, длительным нахождением за компьютером, экологическим прессингом, а также неадекватным питанием. Большую коррекционную роль в решении указанной проблемы призваны сыграть полиненасыщенные жирные кислоты (в частности, класса $\left.\omega_{3}\right)$, входящие в состав современных витаминно-минеральных комплексов для детей. В сообщении описаны технологические аспекты производства одного из таких комплексов, дана оценка сбалансированности состава и концентраций включенных в него нутриентов, а также приводятся примеры клинического опыта его применения у детей с признаками минимальной дисфункции мозга.

Ключевые слова: полиненасыщенные жирные кислоты, витаминно-минеральные комплексы, рациональная фармакотерапия, педиатрия.

(Вопросы современной педиатрии. 2013; 12 (1): 196-198)

Проблема нарушений интеллектуального развития детей в последнее время привлекает все большее внимание не только психоневрологов, но и педиатров. Во всех странах мира, по данным экспертов Всемирной организации здравоохранения, только грубые нарушения умственного развития имеют $15 \%$ детей.

В отношении Российской Федерации можно говорить о значительных умственных перегрузках детей в условиях школьной программы. Кроме того, серьезную опасность представляет собой неконтролируемое время, которое дети проводят у телевизора, за компьютером, пользуясь сотовыми телефонами. Не секрет, что дети недополучают эссенциальные нутриенты с пищей, предпочитая монотонизацию рациона и фаст-фуд. Не снижающееся экологическое загрязнение усугубляет данную ситуацию.

Установлена значимая роль полиненасыщенных жирных кислот (ПНЖК) в усилении адаптации, стимуляции когнитивных способностей, стабилизации нервной системы ребенка [1]. Наиболее ценными ПНЖК принято считать кислоты класса $\omega_{3}$ (линолевая, арахидоновая, докозапентаеновая) и $\omega_{6}$ ( $\alpha$-линоленовая, эйкозапен-

A.A. Trubnikov, S.V. Onegin, S.V. Talashova

Yaroslavl State Medical Academy, Russian Federation

\title{
Usage of modern vitamin-mineral complexes as supplementary source of essential polyunsaturated fatty acids in pediatric practice
}

The prevalence of childhood neurological disorders associated with significant mental overloads, prolonged work with computers, intensified ecological pressure and inappropriate nutrition has been increasing recently. Polyunsaturated fatty acids (particularly $\omega_{3}$ ) included into the composition of the modern vitamin-mineral complexes for children are to play the main corrective role in solving of this problem. Technological aspects of one of such complexes production process and assessment of its composition balance and nutrients' concentrations are described in this article. The authors also give clinical cases of this medicine usage in children with symptoms of minimal cerebral dysfunction.

Key words: polyunsaturated fatty acids, vitamin-mineral complexes, rational pharmacological therapy, pediatrics.

(Voprosy sovremennoi pediatrii - Current Pediatrics. 2013; 12 (1): 196-198) 
таеновая, докозагексаеновая). Эссенциальными жирными кислотами считаются линолевая и $\alpha$-линоленовая, т. к. организм современного человека, в т. ч. и ребенка, утратил способность к их синтезу. Также они являются предшественниками длинноцепочечных ПнЖК (ДЦ ПНЖК), таких как докозагексаеновая (ДГК), арахидоновая (АК) и эйкозапентаеновая (ЭПК) кислоты, которым приписывают основную терапевтическую активность [2].

$\omega_{3}$-ПНЖК оказывают существенное влияние на умственное развитие и зрительные функции ребенка, поскольку являются обязательным компонентом клеточных мембран тканей мозга и сетчатки глаза; составляют основу клеточных мембран, обеспечивая их необходимую проницаемость; регулируют поступление и обмен веществ в клетках.

Недостаток $\omega_{3}$-ПНЖК может приводить к снижению уровня интеллекта, иммунитета и адаптационных возможностей ребенка. Вот почему важно контролировать и своевременно восполнять их дефицит.

Дефицит или дисбаланс ДЦ ПНЖК могут способствовать появлению и развитию часто встречающихся нарушений неврологического развития у детей (синдром гиперактивности и дефицита внимания, дислексия, нарушения развития или диспраксия) и нарушениям, входящим в спектр проявлений аутизма. Введение ДЦ ПНЖК в рацион детей, имеющих синдром гиперактивности с дефицитом внимания и нарушения развития, может уменьшить выраженность симптоматики и обеспечить лучшие показатели в учебе [3].

Интерес к $\omega_{3}$ связан не только с дефицитностью поступления в организм ребенка их с пищей, но и с тем, что происходит замещение недостающих ДЦ ПНЖК производными неэссенциальных жирных кислот семейства $\omega_{9}$, которые могут синтезироваться в организме, или производными, принадлежащими к другим семействам [4]. Эти изменения в конечном итоге могут вести к серьезным нарушениям функций иммунной системы, внутренних органов, зрения, репродукции и др. В связи с этим достаточное поступление с пищей ДЦ ПНЖК является важным условием поддержания здоровья детей. Необходимо не допускать развития дефицитных состояний, связанных с недостаточным поступлением в организм эссенциальных жирных кислот [5].

Приведенные выше факты свидетельствуют о необходимости коррекции рациона питания детей. Одним их вариантов является прием современных витаминноминеральных комплексов, содержащих $\omega_{3}$ ПНЖК в концентрациях, соответствующих возрастным нормам.

Фармацевтическая компания "KRKA" (Словения) выпускает современные витаминные и витаминно-минеральные комплексы для детей в возрасте от 1 до 14 лет. Все они производятся на заводе по производству твердых фармацевтических форм “Нотол". Данное производство отличает технология вертикальной подачи материалов, представляющая собственную разработку компании, что обеспечивает максимальную чистоту и высокое качество готовой продукции. Продукция соответствует всем международным стандартам качества, предъявляемым к фармацевтическим товарам: GMP, ISO серии 9000 и OHSAS 18001:1999.
Линейка "Пиковит" включает 8 наименований разного состава, форм выпуска, для разновозрастной категории детей и постоянно пополняется "новинками" (к примеру, Пиковит Омега 3). Описываемый витаминный комплекс сбалансирован по составу и концентрациям входящих в его состав нутриентов в соответствии с возрастными потребностями детского возраста. Он состоит из 10 необходимых витаминов и $\omega_{3}$-ПНЖК (ЭПК и ДГК, суммарно 205 мг). Известно, что рекомендуется соотношением ЭПК/ДГК в пределах 5/1-3/1 [1]. Именно поэтому в составе данного комплекса соотношение ЭПК/ДГК составляет 125 к 80 мг.

За счет наличия в составе $\omega_{3}$-ПНЖК поливитаминный комплекс обеспечивает благоприятное воздействие на развитие мозга, скорость притока энергии, необходимой для передачи импульсов от клетки к клетке. Его применение позволяет повысить мыслительные способности и сохранить в памяти информацию; способствует оптимальному формированию зрения, а также обеспечивает противовоспалительное и защитное действие.

В связи с тем, что $\omega_{3}$-ПНЖК участвуют в процессах перекисного окисления липидов, их желательно принимать одновременно с антиоксидантами [5]. В составе описываемого витаминно-минерального комплекса присутствуют антиоксиданты - витамины А, Е и С.

Кроме того, в комплексе присутствуют витамины группы $\mathrm{B}\left(\mathrm{B}_{1}, \mathrm{~B}_{2}, \mathrm{~B}_{6}, \mathrm{~B}_{12}\right)$, которые принимают участие в обмене веществ и энергии; способствуют нормальному развитию центральной и периферической нервной системы, а также благоприятно влияют на усиление когнитивных функций. В состав включен и витамин D, принимающий участие в метаболизме кальция и регулирующий содержание фосфора в организме.

Клиническая эффективность данного комплекса витаминов продемонстрирована в исследовании, выполненном на базе детской поликлиники КП "Херсонская городская клиническая больница им. А.С. Лучанского" в отношении комплексного лечения детей раннего возраста с минимальной дисфункцией мозга. Под наблюдением находилась группа из 46 детей на протяжении 2011 г. На основании полученных данных сделан вывод, что на фоне комплексного лечения минимальной дисфункции мозга с добавлением указанного поливитаминного комплекса отмечена следующая положительная динамика - улучшение общего состояния, когнитивных функций, эмоциональной сферы, поведения; нивелирование рассеянной неврологической симптоматики; улучшение функциональной активности головного мозга (на электроэнцефалограмме). Сделано заключение, что поливитаминный комплекс является эффективным и безопасным средством в комплексном лечении детей с различными проявлениями минимальной дисфункции мозга [6].

Таким образом, приведенные выше результаты исследований позволяют говорить о том, что ПНЖК являются ценным терапевтическим средством коррекции когнитивных нарушений у детей и подростков. В свою очередь, Пиковит Омега 3 удовлетворяет всем требованиям, предъявляемым к современным витаминно-минеральным комплексам для детей, имея сбалансированный состав по входящим нутриентам и их концентрациям. 


\section{REFERENCES}

1. Kon I.Ya., Shilina N.M. Omega 3-polyunsaturated fatty acids in prophylaxis and treatment of diseases in children and adults. Lechashhij vrach - Practicing Doctor. 2006; 4: 55-60.

2. Biochemistry. Edited by Severin Ye.S. Moscow: GEOTAR-Media. 2004. C. $417-426$.

3. Richardson A. J. Food and behaviour research. 2007.

4. Titov V.N. Biological substantiation of polyunsaturated fatty acids of $\omega_{3}$ family in prophylaxis of atherosclerosis. Question of medical nutrition. 1999; 68 (3): 34-41.
5. Kon I.Ya., Shilina N. M., Volfson S. B., Georgiyeva O.V. The use of polyunsaturated fatty acids in healthy children's nutrition. Lechashhij vrach - Practicing Doctor. 2013.

6. Zhivilo L.M. Minimal brain dysfunction: the topicality of a problem, optimization of treatment. Voprosi sovremennoi pediatrii Current Pediatrics. 2012; 2 (42): 1-3. 